\title{
Modelagem conceitual de bancos de dados espaci- ais para análise geotécnica
}

\author{
Amorim, J. L. G. \\ Escola Politécnica de \\ Pernambuco \\ Universidade de Pernam- \\ buco \\ 50.720-001 - Recife, Bra- \\ sil
}

\author{
Moura, A. R. L. U. \\ Escola Politécnica de Per- \\ nambuco \\ Universidade de Pernam- \\ buco \\ 50.720-001 - Recife, Brasil
}

\author{
Gusmão, A. D. \\ Escola Politécnica de Per- \\ nambuco \\ Universidade de Pernam- \\ buco \\ 50.720-001 - Recife, Brasil \\ gusmao.alex@ig.com.br
}

\author{
Alves, F. P. \\ Escola Politécnica de Per- \\ nambuco \\ Universidade de Pernam- \\ buco \\ 50.720-001 - Recife, Brasil
}

Resumo As informações referentes ao espaço geográfico são necessárias às atividades que estão relacionadas à engenharia, pois interferem no desempenho e custo de uma obra, e ao meio ambiente, na avaliação de problemas de risco geológico. O conhecimento do solo e suas características, poderá influenciar na escolha do tipo de fundação ou em outras decisões a serem tomadas em relação ao uso e ocupação do solo. Dessa forma, a realização de uma análise espacial em um Sistema de Informações Geográficas $(S I G)$, que integre informações do subsolo aos problemas causados pela ocupação, poderá contribuir para a diminuição do custo das obras, de impactos ambientais e de riscos nos empreendimentos. Este artigo apresenta a modelagem conceitual de dados espaciais, dando ênfase apenas a descrição do diagrama de classes, utilizando o modelo OMT-G, que provê primitivas para modelar a geometria, além de fornecer representações de objetos e relacionamentos espaciais.

\footnotetext{
Abstract Information related to the geographical space are necessary for activities linked to engineering, due to interference on performance and general costs of a construction, as well as the environmental aspects associated to evaluation of geological hazards. The knowledge of soil and its characteristics may influence the choice of a type of foundation or other decisions need to be taken in accordance to the land use and occupation. Thus, the conduction of a spatial analysis on a Geographic Information System (GIS) that integrates subsoil's information and problems generated by land use can contribute to the reduction of constructions' costs, environmental impacts and project risks. This articles presents and conceptual modelling of spatial data, emphasizing the description of the class diagram, using the OMT-G Model, which provides primary information to model the geometry and to provide representations of objects and spatial relationships.
} 


\section{Introdução}

O Município de Recife apresenta uma população de 1.537.704 hab., distribuídos em uma área de $218 \mathrm{~km} 2$ [14], que cresceu sem levar em consideração os condicionamentos do meio físico. Como consequência, trouxe problemas que se caracterizam de risco geológico, tais como deslizamentos, subsidência de aterro, uso de água subterrânea, inundação, erosão marinha e sedimentação [12] [13]. Sua geologia consiste em planície flúvio-marinha, depósitos de mangues, sedimentos flúvio-lagunares e aluviões recentes, que representa um subsolo típico muito variado [1]. Sob o ponto de vista Geotécnico, apresenta grande complexidade, pois é considerada uma das capitais brasileiras mais difíceis de trabalhar o solo e subsolo, por ser uma área de formação sedimentar composta por espessos depósitos de argila mole e ter grande parte de seus espaços sobre aterros [9]. Tais fatores levam ao entendimento de que a prática atual de fundações no Recife é fortemente direcionada pelas características geológico-geotécnicas do subsolo [10] [11].

$\mathrm{Na}$ engenharia, algumas características inerentes ao meio físico podem interferir no desempenho e custo de uma determinada obra. Por isso, o conhecimento do solo e de suas características, irá influenciar na escolha do tipo de fundação ou em outras possíveis decisões a serem tomadas em relação à ocupação do solo. Estudos mostraram que o uso da análise espacial que integra informações do subsolo aos problemas causados pelo uso e ocupação do espaço físico, contribuiu para a diminuição de impactos ambientais e de riscos nos empreendimentos [15] [8] [16].

As análises espaciais são realizadas a partir de um Sistema de Informações Geográficas (SIG), capaz de coletar, armazenar, recuperar dados e oferecer mecanismos para combinar as várias informações, por meio de algoritmos [4], além de armazenar atributos alfanuméricos e geometrias dos diferentes tipos de dados geográficos [6].

A disponibilização e a organização sistemática e periódica dos dados de solo e subsolo da cidade do Recife, armazenados em um SIG, podem facilitar a execução de futuros projetos de fundações, além de minimizar os custos e os riscos nos empreendimentos.

Para a criação de um SIG é importante a etapa de modelagem desses dados, que consiste na abstração de objetos e fenômenos do mundo real, para que possam ser representados adequadamente, em um sistema informatizado.

Nesse contexto, este artigo apresenta como objetivo a modelagem conceitual de dados espaciais, utilizando o modelo OMT-G para definir o diagrama de classes para o banco de dados geotécnico. O modelo OMT-G, descrito pelo Instituto Nacional de Pesquisas Espaciais -INPE [5] [2], utiliza as primitivas da UML e incorpora novas primitivas que permite estruturar banco de dados geográficos. Essa modelagem ajudará na estruturação e definição das operações em um banco de dados, que integrado a um SIG, atuará na gestão da informação geotécnica da cidade do Recife.

\section{Fundamentos Teóricos}

\subsection{Modelagem de dados espaciais}

Um modelo de dados é um conjunto de conceitos usados para descrever a estrutura e as operações em um banco de dados. Dessa forma, a modelagem envolve a abstração dos objetos e fenômenos do mundo real, para obter uma forma de representação simplificada e que seja adequada às finalidades das aplicações do banco de dados [3]. Modelar o mundo real e transportar para um sistema informatizado é uma tarefa complexa, pois envolve a discretização do espaço, como parte do processo de abstração. Nessa etapa, são considerados: (a) diferentes níveis de abstração, onde a realidade é moldada através de conceitos geométricos; (b) a forma como a pessoas percebem o espaço, que depende da experiência e da necessidade específica do observador e escala de representação; (c) natureza diversificada de dados espaciais, que além da geometria, localização no espaço, informações associadas e características temporais, podem prover de origens distintas, a exemplo de dados ambientais, que são derivados de dados de topografia, propriedade do solo etc. e (d) relações espaciais, que são abstrações que ajuda a compreender como os objetos se relacionam com os outros, no mundo real [2].

Para aplicações geográficas, a abstração de conceitos e entidades existentes no mundo real representa uma etapa relevante da implementação de um SIG. Ademais, a eficácia dessa implementação, depende da qualidade da transposição de entidades do mundo real e suas interações, para um banco de dados informatizado [5].

São quatro os níveis de abstração, representados na Fig.1: (a) Nível do mundo real, que contém os fenômenos geográficos reais, como rios, ruas, cobertura vegetal; (b) Nível de representação conceitual, que fornece um conjunto de conceitos com os quais as entidades geográficas podem ser modeladas da forma como são percebidas pelo usuário, em um alto nível de abstração; (c) Nível de apresentação, que oferece ferramentas para especificar os diferentes aspectos visuais que as entidades geográficas podem assumir em aplicações e (d) Nível de implementação, que define padrões, formas de armazenamento e estrutura de dados para implementar cada tipo de entidade [2]. 


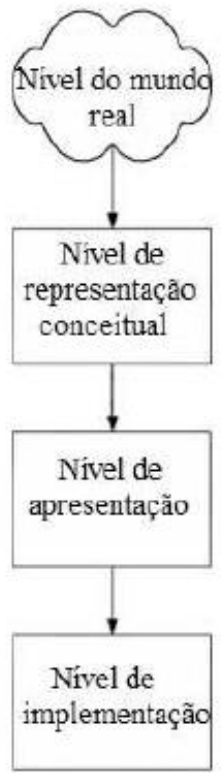

Fig. 1 - Níveis de abstração de aplicações geográficas Fonte: adaptado de [3]

\subsection{O modelo OMT-G}

O OMT-G (Object Modeling Technique for Geographic Applications) é um modelo de dados orientado à objeto, que parte das primitivas definidas para o diagrama de classes da Unified Modeling Language (UML) [18] e introduz primitivas geográficas baseadas nos conceitos básicos de classes, relacionamentos e restrições de integridade espacial, para melhorar a capacidade de representação [2]. Atua nos níveis de representação conceitual e de apresentação (Fig. 1).

O diagrama de classes é usado para descrever a estrutura e o conteúdo de um banco de dados espaciais. Nele estão inseridos apenas regras e descrições que definem, conceitualmente, como os dados serão estruturados. As classes definidas pelo modelo OMT-G podem ser georreferenciadas, que descreve um conjunto de objetos representados espacialmente e associados a regiões da superfície da terra; ou convencionais (não-espaciais), que não possuem ropriedades geométricas, porém, apresentam comportamentos, relacionamentos e alguma relação com os objetos espaciais [2]. A distinção entre ambas as classes permite o compartilhamento de dados não espaciais em aplicações diferentes, facilitando a integração e reutilização de dados [7].

A classe georreferenciada pode ser do tipo geocampo, que representam objetos e fenômenos distribuídos espacialmente de uma forma contínua [7], e do tipo geo-objeto, quando representam objetos geográficos individualizados, que fazem parte do mundo real. O modelo OMT-G define cinco classes descendentes de geo-campo: isolinhas (linhas onde todos os pontos que as compõem possuem o mesmo valor da variável contínua), subdivisão planar (a área de estudo é dividida em regiões segundo um critério de classificação), tesselação (a área é subdividida em células de igual tamanho, na qual é realizada a medição da variável), amostras (representam pontos amostrais da variável contínua) e malha triangular TIN (a partir de isolinhas ou amostras é gerada uma superfície interpolada composta por triângulos irregulares) [17].[2].

São definidas duas classes descendentes de geoobjetos no OMT-G: com geometria e com topologia. A classe do tipo geo-objeto com geometria refere-se à objetos apenas com propriedades geométricas, com classes representadas por ponto, linha e polígono. Já a classe geo-objeto com geometria e topologia, possuem propriedades geométricas e de conectividade topológica, específica para representação de estruturas em redes, e suas especializações são denominadas arco unidirecional, arco bidirecional e nó de rede [2].

Existem várias possibilidades de conceito para uma mesma entidade do mundo real. A exemplo do relevo, que pode ser representado espacialmente por amostras (pontos cotados), por isolinhas (curvas hipsométricas) ou por tesselação (matriz de pixel obtida por interpolação) [17]. Na representação dos conceitos sobre o mundo real no SIG, as entidades podem estar no formato vetorial (define pontos, linhas e áreas por conjuntos de coordenadas) ou matricial (área é dividida em uma grade de células regulares pixels). Os atributos e as operações são especificados para cada classe, no interior do retângulo, conforme apresentado na Fig. 2.



Fig. 2 - Notação gráfica para as classes do modelo OMT-G [2].

O modelo OMT-G considera as relações espaciais e não espaciais na modelagem do espaço. São representados três tipos relacionamentos entre as classes: associações simples (relacionamentos estruturais entre objetos de clas- 
ses diferentes, convencionais ou georreferenciadas); relacionamentos espaciais (relações topológicas, métricas, de ordem e fuzzy) e relacionamentos topológicos em rede (entre objetos que estão conectados uns com os outros) [2]. Existem as chamadas relações 'explícitas', que devem ser especificadas pelo usuário, para permitir que o sistema armazene e mantenha atualizada uma dada informação [2].

Algumas relações espaciais são possíveis apenas entre determinadas classes, pois dependem da sua geometria. O processo de generalização e especialização definem classes a partir de outras classes. As abstrações de generalização e especialização no modelo OMT-G, se aplicam às classes georreferenciadas e convencionais (nãoespaciais) e seguem a notação na UML, onde um triângulo conecta a superclasse à suas subclasses. Cada generalização pode ter um discriminador associado, que indica qual propriedade ou característica está sendo abstraída pelo relacionamento de generalização [2] [3].

Uma forma especial de associação entre objetos é a agregação, que considera que um objeto é formado a partir de outros. Nesse caso, uma agregação pode ocorrer entre classes convencionais, entre classes georreferenciadas (necessário usar agregação espacial) e entre uma classe convencional e uma georreferenciada. Numa agregação espacial são explicitados relacionamentos "todo-parte", onde são impostas restrições de integridade espacial do objeto agregado e dos sub-objetos [2].

\section{Metodologia}

O método utilizado para a modelagem de dados espaciais foi baseado no modelo conceitual OMT-G descrito por Borges et al. [2], a qual refere-se às entidades envolvidas dentro de um projeto específico e seus relacionamentos. A importância de um modelo de dados é ter um entendimento mútuo a respeito dos dados, que posteriormente vão estar organizados dentro de um Sistema Gerenciador de Banco de Dados (SGBD). É no nível de implementação, onde são encontradas as linguagens de definição de dados associadas a SGBD espaciais. Apesar de não ser abordado o nível de implementação neste trabalho, é importante enfatizar que a modelagem conceitual apresenta a vantagem de ser independente do software SIG e do SGBD, que serão utilizados numa etapa posterior, referente a construção do banco de dados espaciais (conjunto de dados inter-relacionados) e sua implantação no SIG.

\subsection{Identificação das entidades e relaciona- mentos espaciais}

Para a gestão da informação geotécnica do município do Recife, foi realizado o levantamento dos dados espaci- ais e não espaciais que serão importantes para serem representados no SIG. A partir da análise de documentos disponíveis, tornou-se possível:

- selecionar os objetos e fenômenos (entidades) do mundo real, que irão constituir as classes de objetos;

- definir as representações dessas entidades espaciais e como estas serão visualizadas, de acordo com o modelo OMT-G;

- identificar os tipos de relacionamentos existentes entre as classes de objetos, especialmente, o relacionamento espacial, a agregação espacial e a generalização espacial e convencional.

Os dados de perfis geotécnicos (tipo de solo, NSPT), executados para cada edificação e dados de fundação (tipo e solução de fundação) foram repassados pela empresa Gusmão Engenheiros Associados Ltda. Dados referentes às ortoimagens e à base cartográfica dela obtida, assim como o Modelo Digital do Terreno, foram cedidas pela Prefeitura Municipal do Recife (PMR). Já o mapeamento geológico foi fornecido pelo Serviço Geológico do Brasil (CPRM).

\subsection{Diagrama de classes}

As classes de objetos foram definidas a partir da lista de entidades espaciais. Foi elaborado o diagrama de classes com as descrições que definem conceitualmente como os dados serão estruturados, incluindo a informação do tipo de representação que será adotado para cada classe, baseado nas primitivas do modelo OMT-G, conforme apresentado na Fig. 3.

Nesta modelagem não foi incluída a classe geoobjeto com topologia, por não haver necessidade de representar a localização geográfica e conectividade entre objetos, no caso específico desse estudo. Nas classes geo-campo e geo-objeto com geometria, cada objeto poderá ou não ter atributos não espaciais associados.

\section{Resultados}

Como o modelo OMT-G é baseado na orientação à objeto, toda a informação geográfica pertencerá a classe de Banco de Dados Geotécnico. De acordo com as classes georreferenciadas, definidas no modelo OMT-G, foram estabelecidas para este estudo as classes que representam a distribuição espacial de uma variável contínua, do tipo geocampo, como sendo: TIN (rede triangular irregular) - Modelo Digital do Terreno (MDT); Amostras - perfil geotécnico; Subdivisão planar - os mapas temáticos (Geológico, 
Geotécnico, Uso e ocupação do solo); Isolinha - curvas de nível e Tesselação - ortoimagens (Fig. 4).

A classe do tipo geo-objeto representou os objetos que possuem propriedades geométricas, ou seja, apenas a localização geográfica dos seus limites, e foram descritos como: Ponto - fundação, tipo de fundação, solução de fundação; Linha - hidrografia; Polígono: quadra, lote e edificação (Fig. 5).

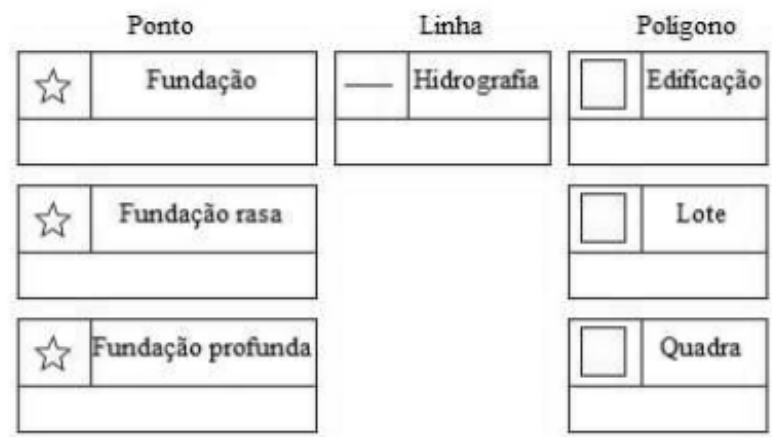

Fig. 5 - Descrição dos geo-objetos sem topologia, estabelecidos.

No modelo OMT-G, os relacionamentos por associações simples são indicados por linhas contínuas, enquanto relacionamentos espaciais são indicados por linhas pontilhadas. este último, indicado na Fig.6. Tal representação facilita a distinção visual entre os relacionamentos baseados em atributos alfanuméricos e baseados na localização e forma geométrica dos objetos [2]. O relacionamento espacial, que considera a relação entre duas classes georreferenciadas, foi levado em consideração na interação entre classes para o caso em que um lote contém edificações (Fig. 6).

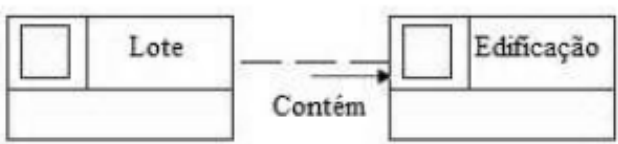

Fig. 6 - Relacionamento espacial entre classes de objeto Edificação e Lote.

Para a classe perfil geotécnico, a relação espacial foi definida por agregação entre classes georreferenciada e convencional (Fig. 7). A espessura da camada de solo, o tipo de solo e valor do NSPT, que constituem as classes convencionais, são atributos da classe georreferenciada, pois representam as características de cada perfil geotécnico.

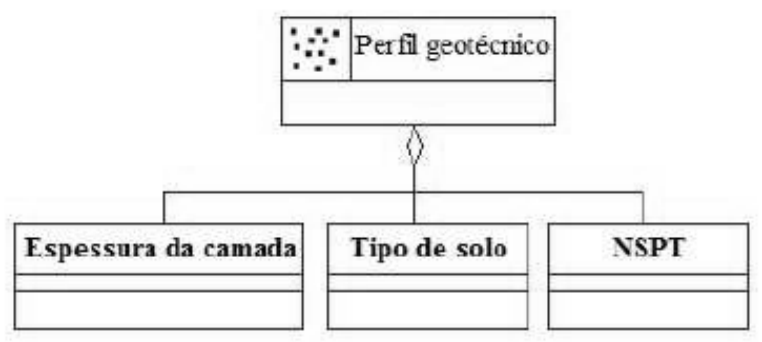

Fig. 7 - Agregação entre classes georreferenciada e convencional.

A agregação entre classes georreferenciadas foi considerada como uma forma especial de associação entre objetos ("todo-parte"), onde a quadra é formada a partir dos lotes (Fig. 8).

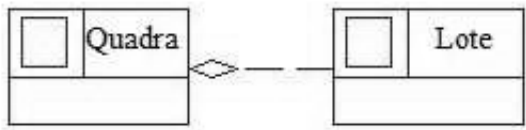

Fig. 8 - Agregação espacial.

Utilizou-se a abstração de generalização espacial para classes georreferenciadas, em que um triângulo conecta a superclasse fundação às subclasses fundação rasa e fundação profunda (Fig. 9). Este processo caracteriza-se pela definição da classe mais genérica (superclasse) a partir de classes com características semelhantes (subclasses). Foi definido um discriminador - tipo de fundação - como indicação das propriedades ou características que estão sendo abstraídas pelo relacionamento. Neste caso, essas características estão representadas pelos dois tipos de fundações, a rasa e a profunda.

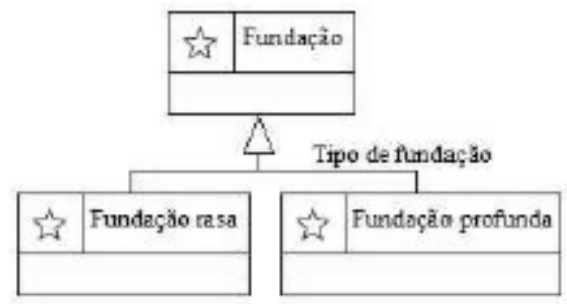

Fig. 9 - Generalização espacial.

Observa-se na Fig.10 que para cada tipo de fundação, foi considerada uma generalização nãoespacial, que utiliza como discriminador - solução de fundação. Esta relação é considerada uma generalização total, pois a união de todas as instâncias das subclasses, representadas pelas diversas soluções de fundação, equivale ao conjunto completo de instâncias da superclasse, representa pelas fundações rasa e profunda. 
As soluções de fundação rasa como sapata (areia e brita, sapata (com argamassa), sapata e radier representam as características que definem os atributos da superclasse fundação rasa. Assim como, estacas prémoldada, hélice contínua, metálica, Franki e raiz, representam os atributos da superclasse fundação profunda.

Como resultado da modelagem conceitual, utilizando o modelo de dados orientado à objeto OMTG, é apresentado o diagrama de classes do Banco de Dados Geotécnico, indicado na Fig. 11.

Os resultados apresentados neste artigo foram gerados utilizando a plataforma UML/MDA StarUML, que consiste em uma ferramenta de modelagem UML, para criar projetos de software, desde os conceitos básicos, até a solução codificada. Nesta ferramenta, o uso do padrão modelo de unidade de Arquitetura (MDA), possibilita uma modelagem complexa [19].

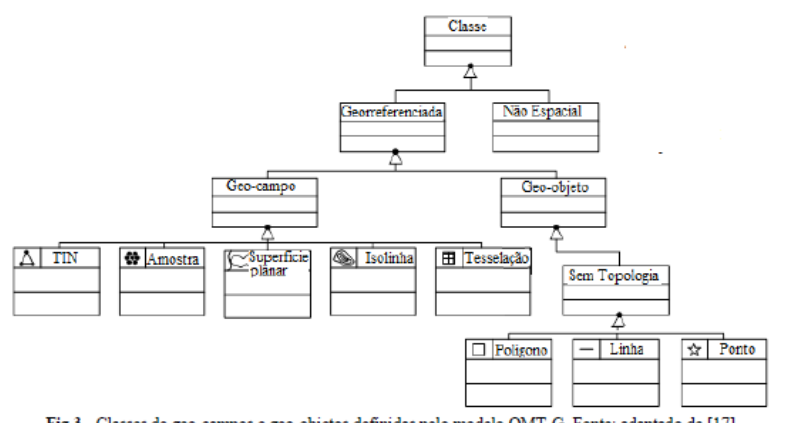

Fig. 3 - Classes de geo-campos e geo-objetos definidas pelo modelo OMT-G. Fonte: adaptado de [17]

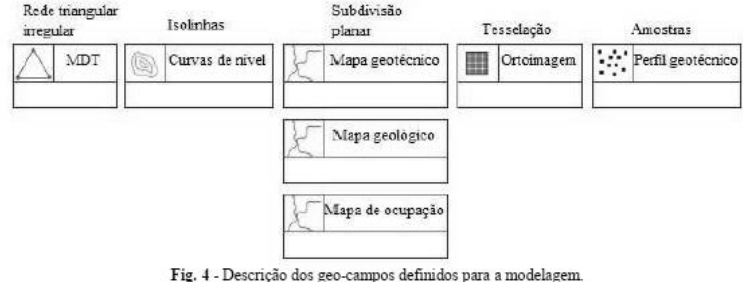

Fig. 4 - Descriç̃o dos geo-campos definidos para a modelagem
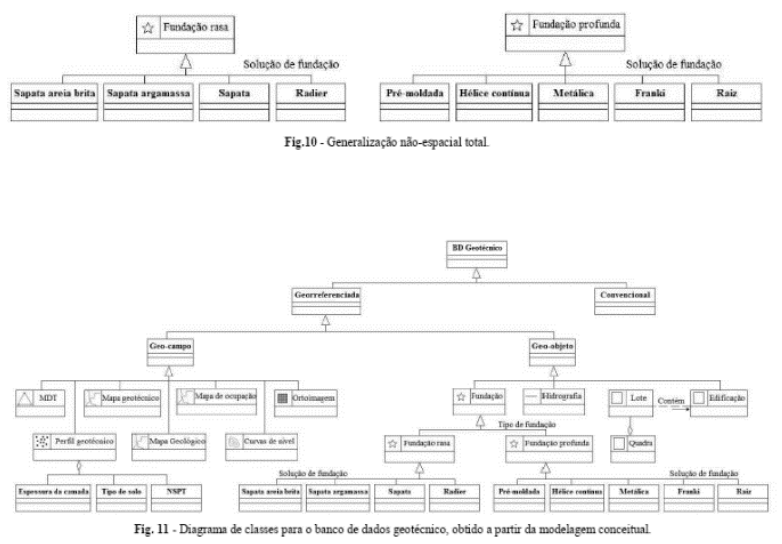

\section{Conclusão}

A importância do modelo conceitual de dados espaciais, dentro do projeto de Sistema de Informações Geográficas, é que todos os envolvidos com o projeto poderão ter a mesma visão da aplicação realizada. A utilização da modelagem conceitual OMT-G, garantiu a homogeneização dos dados espaciais, atendendo aos requisitos de clareza e facilidade de uso para descrever diagrama de classes do banco de dados geotécnico.

Apesar de ser muito complexo abstrair a realidade e moldá-la através de conceitos geométricos, a qualidade da transposição das entidades do mundo real em um alto nível de abstração para um banco de dados informatizado, irá garantir a eficiência da implementação dessas entidades em um SIG.

Este trabalho propôs uma modelagem conceitual dos dados espaciais, dando ênfase apenas a descrição do diagrama de classes. Torna-se portanto, necessária a definição dos diagramas de transformação e apresentação para que seja possível uma estruturação adequada do banco de dados geotécnico.

\section{Referências}

[1] Alheiros, M. M.; Menezes, M.F.; Ferreira, M.G. Carta Geotécnica da Cidade do Recife", Sub- 
Área Geologia/Geologia de Engenharia, Relatório Final de Atividades. FINEP/UFPE, 81 p., 1990.

[2] Borges, K.A.V.; Davis JR, C. A.; Laender, A.H.F. Modelagem conceitual de dados geográficos. Casanova, M.A. et al., Banco de dados geográficos. Curitiba, Mundogeo, p. 93-146, 2005.

[3] Borges, K.A.V.; Davis JR, C.A.; Laender, A.H.F. OMTG: an object-oriented data model for geographic applications, GeoInformatica, v.5, n.3, p. 221-260, 2001.

[4] Burrough, P. A. Principles of Geographical Information Systems for landresources Assessment. Clarendon Press, Oxford, 193p, 1986.

[5] Câmara, G. Representação computacional de dados geográficos. Casanova, M.A. et al., Banco de dados geográficos. Curitiba, MundoGEO, 2005.

[6] Câmara, G.; Medeiros, C. B.; Casanova, M. A.; HEMERLY, A.; MAGALHÃES, G. Anatomia de Sistemas de Informação Geográfica. Escola de Computação, SBC, 1996.

[7] Câmara, G.. Modelos, linguages e arquiteturas para banco de dados geográficos. São José dos Campos: INPE, 1995. Tese de doutorado, 1995.

[8] Diniz, N. C. Cenários de Geoindicadores para Avaliação Ambiental em SIG e Base de Dados Geoambientais. In: Indicadores de sustentabilidad para la industria extractiva minera, Carajás, 2002 .

[9] Gusmão, A. D.; Coutinho, R. Q.; Maia, G. de B.; Oliveira, P. E. S.; Amorim, M. D.; Silva, L. I.; Rolim, M.; Barbosa, V.; PEZO, O. B. Estudo Comparativo de Métodos de Melhoramento de Solos no Recife. In: SEFE7 - Seminário de Engenharia de Fundações Especiais e Geotecnia, 2012, São Paulo. SEFE7 - Seminário de Engenharia de Fundações Especiais e Geotecnia, 2012.

[10] Gusmão, A. D. Prática de fundações no Recife. In: Geotecnia no Nordeste. 1ed. Recife: Ed. Universitária UFPE, v. 1, p. 225-246, 2005.

[11] Gusmão Filho, J. A. Solos - Da Formação Geológica ao Uso na Engenharia. 01. ed. Recife: Ed. Universitária UFPE, v. Único. 198p., 2002.
Ocupadas do Recife: Integração Técnica, Institucional e Comunitária. Rev. IG. São Paulo, Volume Especial p. 9-22, 1995.

[13] Gusmão Filho, J. A. O risco Geológico no Recife. ln: Congresso Brasileiro de Geologia de Engenharia, $7^{\circ}$, Poços de Caldas, MG, ABGE. Mesa Redonda sobre risco geológico, Anais. V. 3 (no prelo), 16p, 1993.

[14] IBGE. Instituto Brasileiro de Geografia e Estatística. Projeto Mudança do Referencial Geodésico - PMRG. Disponível em: <http://www.ibge.gov.br>. Acesso em 01 set. 2014.

[15] Pina, B. e Jeremias, F. Aplicação de um SIG à Informação Geológico-geotécnica obtida no reconhecimento do local do NAL-CTA. E-Terra, Revista Electrónica de Ciências da Terra, Volume 22 $-\mathrm{n}^{\mathrm{o}} 8 \mid 2010$.

[16] Prandini, F. L.; Nakazawa, V. A.; Freitas, C. G. L.; Diniz, L. C. Cartografia Geotécnica nos planos diretoresregionais e municipais. In: Curso de Geologia Aplicada aoMeio Ambiente, ABGE/IPT, São Paulo, ABGE, p.187-202, 1995.

[17] Ramos, J. A. S. Banco de Dados Geográficos.Minicurso. MundoGEO, 2014.

[18] Rational Software Corporation, 1997, The Unified Modeling Language: notation guide, version 1.1 .

[19] StarUML. Site da Internet: http://staruml.software. informer.com/5.0/, visitado pela última vez em 18/09/2014 